\title{
Evaluating the Perception Among Rheumatologists of Maintenance of Board Certification in the US
}

\author{
Amr H. Sawalha and Patrick Coit
}

Objective. There continues to be a debate about the value and purpose of maintenance of certification (MOC) programs in the US. The goal of this study is to assess the impact, value, and purpose of MOC programs in rheumatology.

Methods. A survey was sent to 3,107 rheumatologists in the US. The survey addressed how rheumatologists perceive the value and impact of MOC programs on rheumatology practice and patient care.

Results. A total of 515 rheumatologists completed this survey. The majority $(74.8 \%)$ believed there was no significant value in MOC, beyond what is already achieved from continuing medical education. Most rheumatologists did not believe MOC was valuable in improving patient care (63.5\%), and the majority felt that the primary reason for creating MOC was either the financial well-being of board-certifying organizations (43.4\%) or to satisfy administrative requirements in health systems $(30 \%)$. Although $65.6 \%$ perceived that staying current with new medical knowledge was a positive impact of MOC programs, the MOC was perceived to result in time away from providing patient care (74.6\%) and time away from family (74\%). When asked about potential effects of requiring MOC, $77.7 \%$ reported physician burnout, $67.4 \%$ early physician retirement, and $63.9 \%$ anticipated an effect on reducing the overall number of practicing rheumatologists.

Conclusion. The majority of rheumatologists do not believe there is significant value for MOC programs. There is evidence for lack of trust in board-certifying organizations, and rheumatologists believe MOC programs contribute to physician burnout, early retirement, and loss in the rheumatology workforce.

\section{INTRODUCTION}

Board certification in medical specialties in the US was initially introduced at the turn of the twentieth century to provide a mechanism to demonstrate the clinical competence of practicing physicians. Achieving board certification for a specific specialty or subspecialty used to be a landmark life-long accomplishment for physicians after completing their initial medical training. Board certification in the US is overseen by the American Board of Medical Specialties (ABMS), an umbrella self-governing organization that includes specialty board members such as the American Board of Internal Medicine (ABIM) and the American Board of Pediatrics. Over time, organizations entrusted by physicians to conduct and issue board certifications ended life-long certification, and began issuing time-limited board certificates that required regular renewals. Although ensuring the continuous medical education of physicians was suggested to be the motive for this change, this move

Amr H. Sawalha, MD, Patrick Coit, MPH: University of Michigan, Ann Arbor.

Address correspondence to $\mathrm{Amr} \mathrm{H}$. Sawalha, MD, Division of Rheumatology, University of Michigan, 1150 W. Medical Center Drive remains controversial because a benefit to patients by this process has not been supported by credible scientific, non-conflicted data, and because it excluded or "grandfathered" physicians who had received their initial board certification at an arbitrary earlier time point (1).

More recent changes in board recertification requirements and the expansion of a controversial maintenance of certification (MOC) program finally caught the public attention and resulted in significant discussions both at the national level and within the medical community concerning the value, motive, and consequences behind this program and board recertification $(2,3)$. It has been suspected that this program financially supports board-certifying organizations that have been growing in size, and although self-declared as non-profit organizations, have actively participated in lobbying activities $(4,5)$. Interestingly, MOC programs operated by a specialty body of the ABMS were included as a measure for physician quality and payment incentives within

5520 MSRB1, SPC 5680, Ann Arbor, MI 48109. E-mail: asawalha@umich. edu.

Submitted for publication August 22, 2018; accepted in revised form December 4, 2018. 


\section{SIGNIFICANCE \& INNOVATIONS}

- The survey assessed the value of a maintenance of board certification (MOC) program from the perspective of rheumatologists in the US.

- Overall, there appears to be no perceived significant value for MOC among rheumatologists.

- $\mathrm{MOC}$ is perceived to contribute to a shortage in the rheumatology workforce in the US, without proven benefit to patients.

- There is evidence for lack of trust among rheumatologists in board-certifying organizations. A financial conflict of interest in creating MOC is perceived to be a likely reason for creating MOC among rheumatologists.

the Affordable Care Act $(6,7)$. Non-transparent and at times questionable financial practices of board-certifying organizations have surfaced in the public domain, eroding trust in these organizations of physicians and medical societies (4). In response, apologies and restructuring attempts have been made (8), but the core issue of whether recertification and $\mathrm{MOC}$ programs positively impact health care has remained unclear $(9,10)$. It is alarming that while no clear noncontroversial benefit to patients from $\mathrm{MOC}$ has been demonstrated, the mean estimated cost of the ABIM MOC program for all internal medicine physicians is estimated to be $\sim \$ 5.7$ billion over a 10-year period, of which $\$ 561$ million are fees payable to the ABIM (11).

Board certification, which started as a voluntary achievement and remains so in theory, has become involuntary in practice, making participation in $\mathrm{MOC}$ programs mandatory for many if not most physicians in order to maintain employment and clinical privileges, or receive reimbursement. The controversy surrounding the motive behind creating MOC, the value of MOC, and the fear for this requirement to interfere with the ability to practice, has prompted legislatures in several states to prohibit using MOC as a condition for employment, licensure, securing clinical privileges, and reimbursement. Oklahoma was the first state to pass such legislation. The National Board of Physicians and Surgeons (NBPAS) was established as a grass-roots organization to provide an alternative recertification process that would allow physicians to recertify to maintain their practices at a fraction of the cost required by the ABMS and ABIM. To date, NBPAS, which uses participation in continuing medical education (CME) activities as a basis for recertification, has certified over 7,000 physicians and is accepted by 90 hospitals and health systems within the US (12).

Rheumatologists have been very closely following the controversy regarding $\mathrm{MOC}$ and board recertification. Indeed, the American College of Rheumatology (ACR) has questioned the value of the ABIM MOC program, and issued a statement raising concerns regarding the cost of $\mathrm{MOC}$, lack of evidence to support beneficial impact of MOC on clinical care, and concerns about the financial stewardship of the ABIM (13). The goal of this survey study was to assess the current perception of practicing US rheumatologists of the value of board recertification and MOC programs, and assess the impact of these programs on rheumatologists and the rheumatology workforce.

\section{MATERIALS AND METHODS}

A survey was designed to assess the impact and perceived value of MOC programs in rheumatology. The survey consisted of 20 questions, including 19 closed-ended questions and 1 open-ended question (Supplementary Appendix 1, on the Arthritis Care \& Research web site at http:// onlinelibrary.wiley.com/doi/10.1002/acr.23823/abstract). The questions were designed to address issues relevant to concerns discussed in recent editorials, opinion pieces, and medical society statements concerning $\mathrm{MOC}$ programs $(2-5,13)$. The 2 questions pertaining to the perceived positive and negative impact of board recertification and MOC were randomized such that half of the respondents receive each of the 2 questions first. The survey included 1 ranking question, with the order of possible answers for this question also being randomized.

Ten practicing rheumatologists at the University of Michigan, (including a mixture of predominantly clinical faculty and physician scientists who are ABIM board certified and participating in

Table 1. Demographics, primary specialty, and primary work setting of respondents*

\begin{tabular}{lcc}
\hline & No. & Frequency, \% \\
\hline Primary specialty & & \\
Adult rheumatology & 469 & 91.2 \\
Pediatric rheumatology & 33 & 6.4 \\
Both adult and pediatric & 12 & 2.3 \\
Primary work setting & & \\
Academia/university & 223 & 43.4 \\
Private practice & 231 & 44.9 \\
Government & 19 & 3.7 \\
Industry/pharmaceutical & 3 & 0.6 \\
Other & 38 & 7.4 \\
Age, years & & \\
30-39 & 29 & 5.6 \\
40-49 & 160 & 31.1 \\
50-59 & 142 & 27.6 \\
$60-69$ & 152 & 29.6 \\
$\geq 70$ & 31 & 6.0 \\
Sex & & 39.3 \\
Female & 202 & 60.7 \\
Male & 312 & \\
\hline
\end{tabular}

* Demographic information was provided by 514 of 515 survey respondents. 


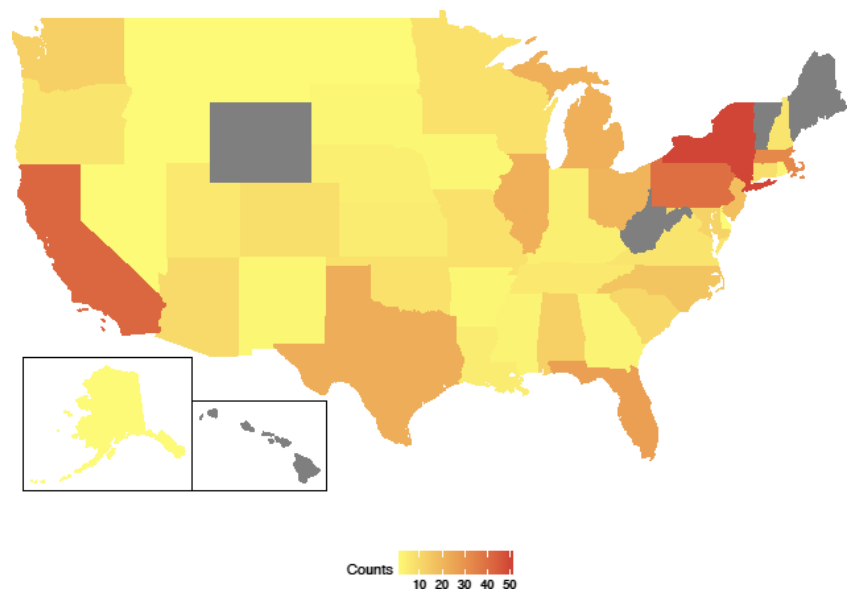

Figure 1. The geographic distribution of rheumatologists who responded to the survey.

$\mathrm{MOC}$ ) were asked to provide input on the survey questions before the questions were finalized. These 10 rheumatologists and the authors were excluded from taking the survey for the purpose of this study. The survey design and all survey questions were reviewed by staff at the Consulting for Statistics, Computing \& Analytic Research at the University of Michigan. The survey was constructed using SurveyMonkey (San Mateo, CA) and sent via email to 3,107 rheumatologists within the US. Invitations to participate in this survey were initially sent out to all 3,107 rheumatologists on March 6, 2018 with responses collected until March 26, 2018 when participation in this survey was closed. Analysis of survey results was performed in the computing environment $\mathrm{R}$.

\section{RESULTS}

A total of 515 rheumatologists completed the survey. With an estimated number of $\sim 5,000$ practicing rheumatologists in the US, this sample size provides 95\% confidence that the expected responses of practicing rheumatologists in the US are within a margin of error of less than $5 \%$ of the responses obtained from the rheumatologists who completed this survey.

The majority of respondents were adult rheumatologists (91.2\%), while 6.4\% were pediatric rheumatologists, and $2.3 \%$ were both adult and pediatric rheumatologists. The majority of respondents identified their primary work setting as either private practice $(44.9 \%)$ or a university or academic setting (43.4\%). Of the total respondents, $60.7 \%$ were male and $39.3 \%$ were female rheumatologists. In terms of age, the majority of respondents

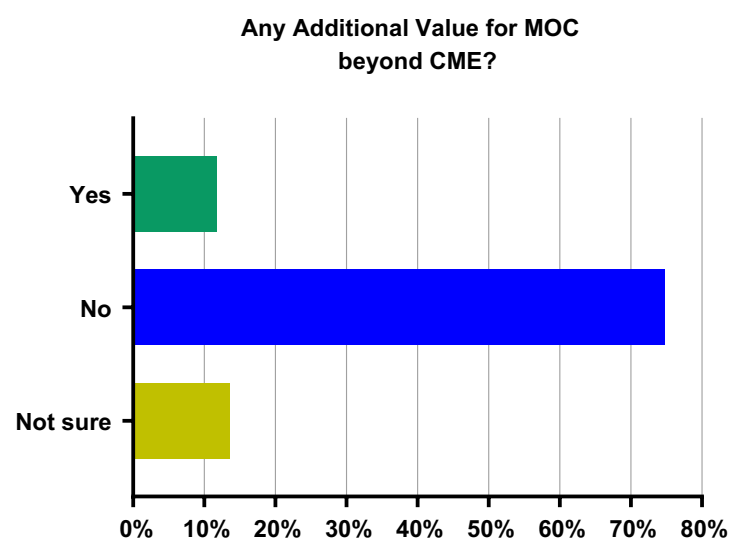

Value of MOC for Improving Patient Care

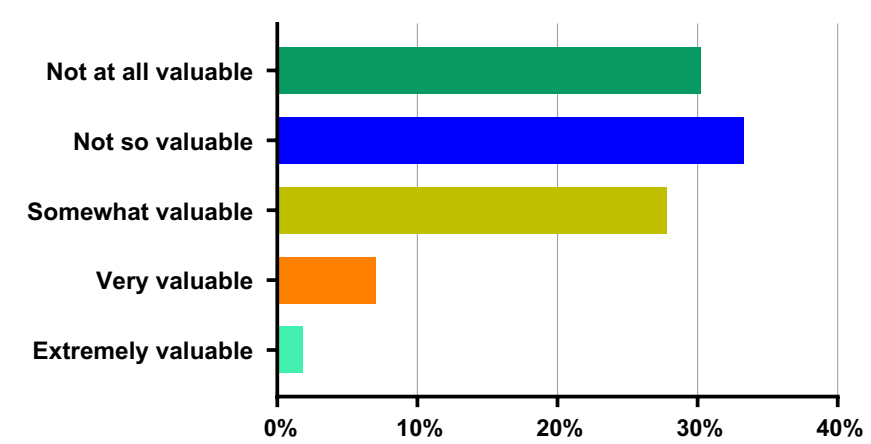

Primary Reason for Creating MOC

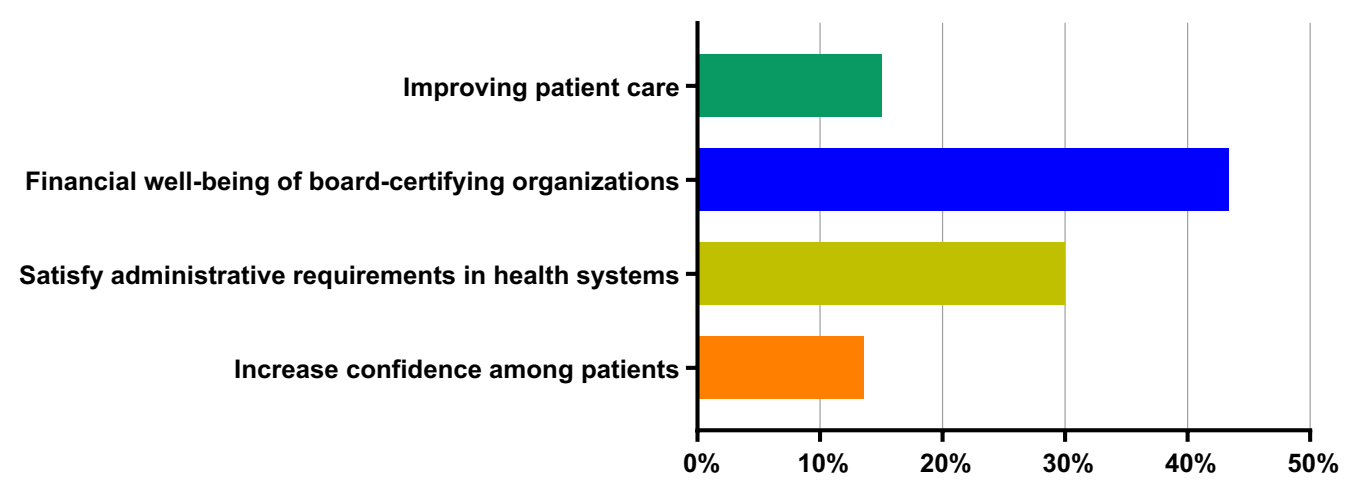

Figure 2. Summary of responses evaluating the value of maintenance of certification (MOC) programs among rheumatologists beyond continuing medical education (CME) and as it pertains to improving patient care, and the perceived reasons for creating MOC programs. 
were either age range $40-49$ years $(31.1 \%)$ or $60-69$ years (29.6\%) (Table 1). Figure 1 shows the geographic distribution for practice locations for the rheumatologists responding to this survey.

The majority of surveyed rheumatologists in the US (74.8\%) did not think there was significant additional value in MOC, beyond what is already achieved from CME. Indeed, $63.5 \%$ of rheumatologists did not believe board recertification and $\mathrm{MOC}$ were valuable in terms of improving patient care (Figure 2). When asked about the primary reason for creating $\mathrm{MOC}$, the majority of rheumatologists felt it was for the financial well-being of board-certifying organizations (43.4\%) or to satisfy administrative requirements in health systems (30\%). Only $15.1 \%$ believed improving patient care was the primary reason for MOC (Figure 2). The majority of rheumatologists believed board certification should be a life-long credential (63.7\%).

Notably, when asked about possible negative impacts of $\mathrm{MOC}$, the majority reported that MOC resulted in time away from providing patient care (74.6\%), time away from family (74\%), and psychological stress (69.7\%). In addition, 88.5\% of rheumatologists believed that $\mathrm{MOC}$ imposes a financial burden on rheumatologists without proven benefits to patients. When asked about possible positive impacts of MOC, 65.6\% perceived staying current with new knowledge as a positive impact. Most rheumatologists did not identify patient reassurance, improved quality of patient care, or increased patient satisfaction as possible positive impacts of MOC. When asked about anticipated effects of requiring MOC, $77.7 \%$ of the respondents reported physician burnout, $67.4 \%$ early physician retirement, and $63.9 \%$ anticipated a reduction in the overall number of practicing rheumatologists. Only $14.2 \%$ of the respondents believed requiring $\mathrm{MOC}$ will improve the overall quality of practicing rheumatologists in the US, and $75.2 \%$ favored legislation in their state to remove $\mathrm{MOC}$ as a requirement for employment, insurance reimbursement, or securing clinical privileges (Figure 3).

Of interest, 58.9\% of the respondents believed board certification in rheumatology should be administered or overseen by other organizations such as the ACR, and $53.7 \%$ of the surveyed rheumatologists reported participation in basic, translational, or clinical research in rheumatology. Of the respondents who reported participating in research activities, 39.6\% believed MOC was adversely affecting their ability to perform research or research-related activities.

The survey included 1 open-ended question, in which rheumatologists were asked to provide any other relevant thoughts or comments. Comments were received from 186 survey respondents. To summarize these responses, individual ideas or thoughts were grouped into categories that summarized the themes discussed. These groups were ranked based on the number of times a theme was mentioned among all responses. The top 5 themes are listed in Table 2. Overall, these comments echoed the results derived from the closed-ended questions in the survey and stressed issues such as questioning the value of MOC over CME activities, the relevance of material covered by recertification examinations to daily rheumatology clinical practice, the high expense associated with $\mathrm{MOC}$, and the motive behind developing MOC programs.

\section{DISCUSSION}

This study was conducted to assess the perception of the value and impact of MOC programs from the perspective of rheumatologists, and how these programs might affect rheumatology practice and the rheumatology workforce in the US. The data derived from this survey study suggest an overall lack of value for these programs, as perceived by prac-
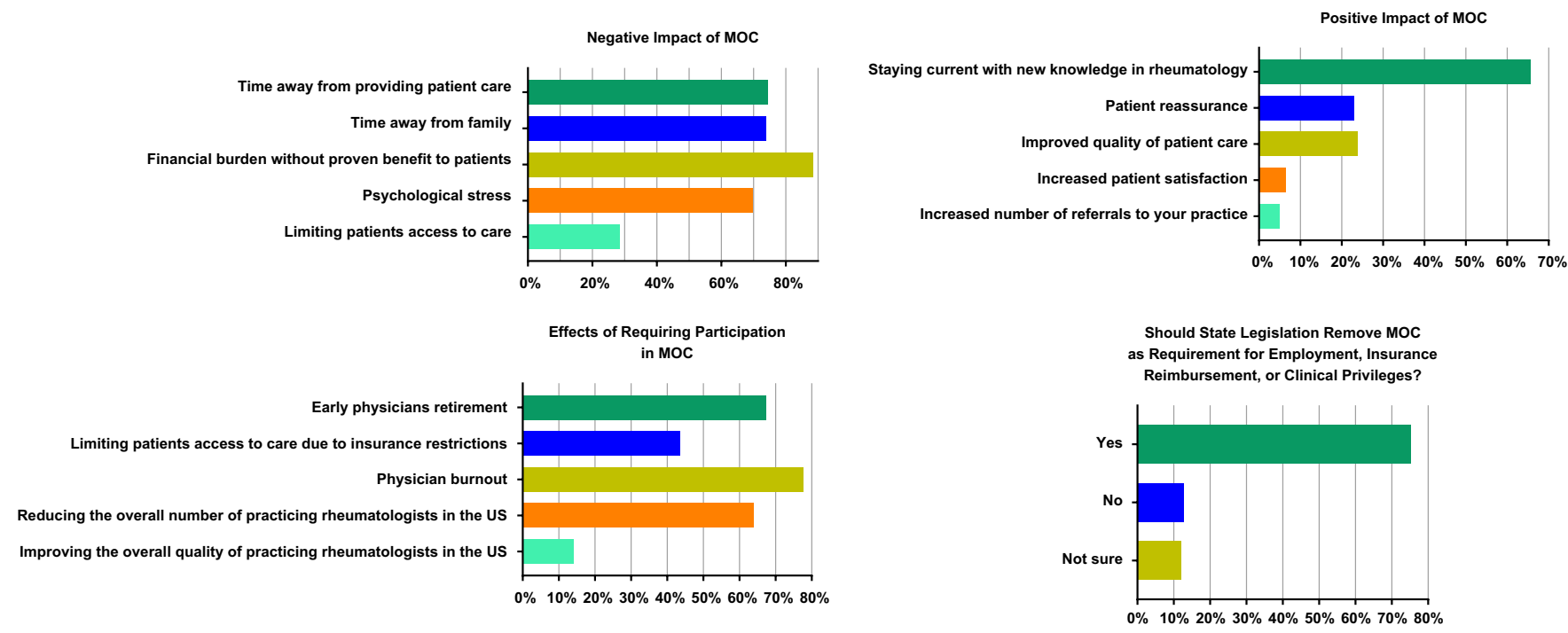

Figure 3. Summary of responses evaluating the impact of maintenance of certification (MOC) programs among rheumatologists. 
Table 2. Top ranked themes described by respondents in the open-ended survey question asking for additional thoughts or comments $^{\star}$

\begin{tabular}{cc}
\hline Rank & Theme \\
\hline 1 & $\begin{array}{c}\text { Critical of monetary and time cost of MOC and/or } \\
\text { impact on patient care }\end{array}$ \\
2 & Critical of the MOC idea and process \\
3 & $\begin{array}{c}\text { CME is sufficient and more relevant than MOC to } \\
\text { stay up to date }\end{array}$ \\
4 & Critical of ABIM and its motives \\
5 & $\begin{array}{l}\text { MOC examination is not relevant to clinical } \\
\text { practice }\end{array}$ \\
\hline
\end{tabular}

* $\mathrm{MOC}=$ maintenance of certification; $\mathrm{CME}=$ continuing medical education; $\mathrm{ABIM}=$ American Board of Internal Medicine.

ticing rheumatologists, which is consistent with other recent studies across different specialties (10). Physicians, including rheumatologists, are committed to life-long learning and appreciate the importance of keeping up to date with recent knowledge and developments in the field to provide the best possible patient care. It seems that the majority of rheumatologists do not believe that MOC programs are the best means to achieve this goal and ensure physician competence. The majority believe that MOC programs do not add significant value to participation in CME activities, which are already required to maintain and renew state medical licensures. Indeed, unlike MOC programs, CME activities can be more flexible and allow individual rheumatologists to participate in educational activities that are most relevant to their individual practices or the patient populations they manage. In addition, CME activities can be achieved at a fraction of the cost required to participate in $\mathrm{MOC}$ programs. A recent cost analysis suggests that the ABIM's MOC is associated with significant testing and time costs for rheumatologists participating in that program, with a mean cost of the program over 10 years of $\$ 21,606$ per rheumatologist, and a mean cost aggregated for rheumatology as a specialty of $\$ 89$ million, of which $\$ 11$ million are fees payable to the ABIM (11).

Importantly, the overwhelming perception of rheumatologists in the US is that enforcing MOC participation results in physician burnout and a reduction in the rheumatology workforce. When asked about whether rheumatologists are required to participate in $\mathrm{MOC}$ for employment or insurance reimbursement, $36.2 \%$ of rheumatologist reported that they are required to participate in MOC and another $23.7 \%$ reported they are not sure if they are required to participate. These data suggest that while the concept of board certification was initially introduced as a voluntary achievement, time-limited certification and now MOC are becoming required for many rheumatologists to sustain employment or practice. The 2015 ACR Workforce Study suggested a current shortage in the rheumatology workforce, and predicted a decline in the number of practicing rheumatologists by 2030 with a supply being outnumbered $\sim 2$-fold by the demand for rheumatologists in the US $(14,15)$. If participation in MOC does not have a significant value on improving patient care in rheumatology, as indicated by the perception of practicing rheumatologists, and if it remains a requirement to practice for at least one-third of rheumatologists in the US, then an argument can be made that elimination of MOC might be a way to sustain and improve the rheumatology workforce without compromising quality. To our knowledge, there have not been any studies to show that rheumatologists participating in MOC activities provide better care.

A striking finding from our study is the indication that there seems to be a lack of trust by practicing rheumatologists in the US in board-certifying organizations and their motives. When asked to rank in order what is thought to be the reason for creating MOC programs, financial well-being of board-certifying organizations was the highest ranked answer. Improving patient care, which is the motive claimed by board-certifying organizations, was the lowest ranked reason. Regardless of what the motive might be, these results suggest that practicing physicians, and in this case rheumatologists, do not trust board-certifying organizations. Therefore, we suggest that these organizations revisit their relationships with practicing physicians, and facilitate true collaboration with those physicians to determine the best way to assess and ensure physician competence and knowledge. Of interest, $\sim 60 \%$ of rheumatologists believe that alternative organizations, such as the ACR, should be involved in administering or overseeing board certification of rheumatologists.

The $\mathrm{NIH}$ has warned that imposing time-consuming MOC programs seems to discourage physician-scientists from maintaining clinical practice (16). This is a potentially serious problem in rheumatology because physician-scientists are the drivers of new discoveries that result in better treatment options and improved care of patients with rheumatologic conditions. Researchers who maintain their own clinical practice are able to stay connected with research questions that are of immediate interest to the diseases being studied and to the patients. In addition, eliminating or reducing the number of physician-scientists who participate in patient care will reduce the number of some of the most qualified and talented physicians who can take care of the most complicated patients in rheumatology and understand the specific disease(s) related to their expertise. Along these lines, our survey revealed that about $40 \%$ of rheumatologists who participate in research report a negative impact of $\mathrm{MOC}$ on their research careers, as reflected by adversely affecting their ability to perform research or research-related activities.

Limitations of our study include the fact that the survey focused on evaluating perception and attitudes of rheumatologists, and the results cannot be interpreted to provide actual consequences of MOC impact. Nonetheless, the rheumatologist's perceptions and opinions captured in this survey should, at the very least, invite a serious independent nonconflicted evaluation of these programs. The survey did not 
address alternative ways for structuring MOC. However, our results indicate that most rheumatologists believe that participating in CME activities is sufficient and they perceive no significant additional value for the MOC program. This belief is consistent with the ACR statement on MOC suggesting that earning MOC points (mandated MOC educational activities) is redundant (13).

In summary, our results suggest that the majority of rheumatologists in the US are concerned about recertification examinations and MOC programs. It appears that these programs are not perceived to be of significant value and do seem to have the potential to contribute to the shortage in the rheumatology workforce in the US. Importantly, there is evidence for eroding credibility and lack of trust in boardcertifying organizations among rheumatologists, and a notion that imposing a time limit on board certification and mandating participation in expensive MOC programs is largely driven by financial interests of these organizations rather than improving patient care. The medical community in general and the rheumatology community in particular need to address the gradual transformation of board certification and MOC from a voluntary activity to practically a requirement in order for many physicians to be able to practice medicine and get reimbursed for services provided. It is important to caution against lobbying activities driven by financial interests in setting health care policies, especially in mandating expensive programs such as $\mathrm{MOC}$, in the absence of convincing data that demonstrate improved patient care, which could result in serious consequences in a field such as rheumatology that is threatened by a large shortage in the workforce.

\section{AUTHOR CONTRIBUTIONS}

Both authors were involved in drafting the article or revising it critically for important intellectual content, and both authors approved the final version to be published. Dr. Sawalha had full access to all of the data in the study and takes responsibility for the integrity of the data and the accuracy of the data analysis.

Study conception and design. Sawalha.

Acquisition of data. Sawalha.

Analysis and interpretation of data. Sawalha, Coit.

\section{REFERENCES}

1. Hayes J, Jacksn JL, McNutt GM, Hertz BJ, Ryan JJ, Pawlikowski SA. Association between physician time-unlimited vs time-limited internal medicine board certification and ambulatory patient care quality. JAMA 2014;312:2358-63.

2. Centor RM, Fleming DA, Moyer DV. Maintenance of certification: beauty is in the eyes of the beholder. Ann Intern Med 2014;161:226-7.

3. Iglehart JK, Baron RB. Ensuring physicians' competence-is maintenance of certification the answer? N Engl J Med 2012;367:2543-9.

4. Fisher WG, Schloss EJ. Medical specialty certification in the United States-a false idol? J Interv Card Electrophysiol 2016;47:37-43.

5. Kempen PM. Maintenance of Certification - important and to whom? J Community Hosp Intern Med Perspect 2013;April 17:3.

6. Kinney ED. The Affordable Care Act and the Medicare Program: linking Medicare payment to quality performance. NYU Annual Survey of American Law. 2014; Research Paper No. 2014-22

7. The Patient Protection and Affordable Care Act; 124 STAT. 119, Public Law 111-148. 2010; URL:https://www.congress.gov/111/ plaws/publ148/PLAW-111publ148.pdf.

8. Baron RJ. ABIM announces immediate changes to MOC Program. URL: https://www.abim.org/news/abim-announces-immediatechanges-to-moc-program.aspx

9. Cook DA, Holmboe ES, Sorensen KJ, Berger RA, Wilkinson JM. Getting maintenance of certification to work: a grounded theory study of physicians' perceptions. JAMA Intern Med 2015;175:35-42.

10. Cook DA, Blachman MJ, West CP, Wittich CM. Physician attitudes about maintenance of certification: a cross-specialty national survey. Mayo Clin Proc 2016;91:1336-45.

11. Sandhu AT, Dudley RA, Kazi DS. A cost analysis of the American Board of Internal Medicine's Maintenance-of-Certification Program. Ann Intern Med 2015;163:401-8.

12. National Board of Physicians and Surgeons (NBPAS). Home page. URL: https://nbpas.org/.

13. American College of Rheumatology. Position Statement maintenance of certification. URL: https://www.rheumatology.org/Portals/O/Files/ ACR\%20MOC\%20Postion\%20Statement\%202015.pdf.

14. Bolster MB, Bass AR, Hausmann JS, Deal C, Ditmyer M, Greene KL, et al. 2015 American College of Rheumatology Workforce Study: the role of graduate medical education in adult rheumatology. Arthritis Rheumatol 2018;70:817-25.

15. Battafarano DF, Ditmyer M, Bolster MB, Fitzgerald JD, Deal C, Bass AR, et al. 2015 American College of Rheumatology Workforce Study: supply and demand projections of adult rheumatology workforce, 2015-2030. Arthritis Care Res (Hoboken) 2018;70:617-26.

16. US Department of Health \& Human Services. Physician-scientist workforce (PSW) report 2014. URL: https://report.nih.gov/ Workforce/PSW/challenges.aspx. 